\title{
Plant growth and their root development after inoculation of arbuscular mycorrhizal fungi in coal mine subsided areas
}

\author{
Yinli Bi ${ }^{1}$ (D) Yanxu Zhang ${ }^{1} \cdot$ Hui Zou ${ }^{1}$
}

Received: 11 August 2017/Revised: 4 March 2018/Accepted: 16 March 2018/Published online: 9 May 2018

(C) The Author(s) 2018

\begin{abstract}
Coal mining often cause serious land degradation, soil erosion, and desertification affecting growth of the local vegetation, especially the roots. Arbuscular mycorrhizal fungi (AMF) inoculation is considered a potential biotechnological tool for mined soil remediation because mycorrhizal fungi could improve plant growth environment, especially under adverse conditions due to their good symbiosis. A field experiment was conducted to study the ecological effects of AMF (Funneliformis mosseae, Rhizophagus intraradices) on the growth of Amygdalus pedunculata Pall. and their root development in the regenerated mining subsidence sandy land. The reclamation experiment included four treatments: inoculation of Funneliformis mosseae (F.m), inoculation of Rhizophagus intraradices (R.i), combined inoculation of F.m and R.i and non-inoculated treatment. Root mycorrhizal colonization, plant height, crown width, soil moisture, root morphology and certain soil properties were assessed. The results showed that AMF improved the shoot and root growth of Amygdalus pedunculata Pall., and significantly increased root colonization after 1 year of inoculation. Available phosphorus content, activities of phosphatase as well as electrical conductivity in soil rhizosphere of all the three inoculation treatments were higher than that of the non-inoculated treatment. AMF increased the quantity of bacteria and fungi in soil rhizosphere compared with the non-inoculated treatment. Our study indicates that revegetation with AMF inoculum could influence plant growth and root development as well as soil properties, suggesting that AMF inoculation can be effective method for further ecological restoration in coal mine subsided areas.
\end{abstract}

Keywords Arbuscular mycorrhizal fungi $\cdot$ Plant growth $\cdot$ Root development $\cdot$ Soil remediation $\cdot$ Amygdalus pedunculata Pall.

\section{Introduction}

China is the world's largest coal producing and consuming country ( $\mathrm{Li}$ et al. 2011). Although coal brings huge economic benefits to China, coal mining causes numerous environmental problems, such as drastic reduction in the number of biological communities in the reconstructed soil induced by any disturbance in coal mining, plant root

Yinli Bi

ylbi88@126.com

1 State Key Laboratory of Coal Resources and Safe Mining, China University of Mining and Technology (Beijing), Beijing 100083, China damage caused by mining subsidence, reduced topsoil fertility, water-fertilizer disharmony in soil, and pollution by filling materials (Zang et al. 2010; Tripathi et al. 2009). Simultaneously, the vegetation coverage is gradually decreasing and thus severely restricts the sustainable development of eco-environment in Western China. Previous studies demonstrated that microbial activity is a key factor affecting the functioning of all terrestrial systems (Quadros et al. 2016).

Soil microbiota communities play crucial role in maintaining the soil ecological balance as well as the sustainability of both natural ecosystem and agro-ecosystem. Beneficial rhizospheric microbe-plant interactions have a great influence on plant health and soil quality (Wu et al. 
2008). Among these beneficial rhizospheric microbes, arbuscular mycorrhizal fungi (AMF) and plant-growth promoting rhizobacteria (PGPR) can be considered. Arbuscular mycorrhizal fungi are obligate symbionts that colonise the roots of most cultivated plant species. Mycorrhizal symbiosis can be found in nearly all types of ecological situations and most plant species are able to form this symbiosis naturally (Gong et al. 2012). These associations occur naturally when plantlets are transplanted into the field, favouring plant development by increasing nutrient uptake, growth rates and hormonal activities (del Carmen Jaizme-Vega et al. 2006; Chandanie et al. 2009). Mycorrhizae may also increase plant tolerance to stress conditions such as salinity and drought (Latef and $\mathrm{He}$ 2011), heavy metals (Tian et al. 2013), root soil borne pathogens (Miller and Jastrow 1992) and the improvement of soil structure (Matthiasc et al. 2010).

Amygdalus pedunculata Pall. is a native tree specie with quick adaptability and high economic and ecological value, which is one of the economic plants used as ecological restoration pioneering species in Shendong coal mine areas (Ma et al. 2006). Studies concerning Amygdalus pedunculata Pall. mainly focus on the physiological and ecological characteristics, plant breeding methods, resources development and utilization, etc., mechanisms and effects of AMF on it has rarely been studied (Ma et al. 2006; Xu and Chu 2013; Chen et al. 2013; Cao et al. 2016). In this study, the impact of two Glomus species on the plant growth and their root development in coal mining subsided land were studied.

\section{Materials and methods}

\subsection{Study site description}

The study was conducted at Daliuta mining area located at the transition zone between Loess Plateau in Northern Shaanxi and the Mu Us Desert. Its altitude ranges from 1000 to $1250 \mathrm{~m}$ and has a semiarid continental monsoon climate. The average annual temperature is about $8.4{ }^{\circ} \mathrm{C}$ with the highest temperature of $38{ }^{\circ} \mathrm{C}$ and the lowest of $-28{ }^{\circ} \mathrm{C}$. Mean annual precipitation is $441.2 \mathrm{~mm}$, which is concentrated between June and September. Annual average evaporation is $2211.2 \mathrm{~mm}$. The area has thick loosened aquifer, and the soil is sandy (Bi and Zhang 2014).

\subsection{Experimental materials}

Two AM fungi were used in the study. Funneliformis mosseae (F.m)was cultured under maize with $90 \%$ of root colonisation, and Rhizophagus intraradices(R.i)was cultured under maize with $84 \%$ of root colonization. They are two kinds of AMF with wide application and strong ability to adapt to the coal mine environment. The tested plant is Amygdalus pedunculata Pall. under field conditions. They were planted in April 2012. For the inoculation treatments, $50 \mathrm{~g}$ of sagent were inoculated near by the root after digging out the root carefully in the following July at the beginning of the nursery phase. After microbial inoculants were evenly applied on the plants, soil was filled back and compacted.

\subsection{Experimental design}

The experiment was conducted by a completely random design method to 4 treatments: (1) non-inoculated as the control treatment(CK); (2) single inoculation of F.m, (3) single inoculation of R.i, and (4) combined inoculation of F.m and R.i. The soil samples were collected in September 2013, 1 year after inoculation. Each treatment was replicated ten times.

\subsection{Experimental methods}

After choosing standard plants randomly in each small quadrat and removing the surface litter around plant roots, the rhizosphere soil in depths of $0-20 \mathrm{~cm}$ was collected into sterile plastic bags, frozen and quickly transported back to the laboratory for analysis. The soil samples were divided into two parts, one was sieved through a mesh of $<2 \mathrm{~mm}$ in diameter, and preserved at $4{ }^{\circ} \mathrm{C}$ for analysis of microbial biomass and enzymatic activity; the other was naturally air-dried, and sieved through a mesh of $<1 \mathrm{~mm}$ in diameter for analysis of physical-chemical properties. Microbial populations were conducted by dilution plate method (Shen et al. 1999). The medium for actinomycesis named improved Gao compound medium, including soluble starch $2 \mathrm{~g}, \mathrm{KNO}_{3} 0.1 \mathrm{~g}, \mathrm{~K}_{2} \mathrm{HPO}_{4} 0.05 \mathrm{~g}, \mathrm{MgSO}_{4} \cdot 7 \mathrm{H}_{2} \mathrm{O}$ $0.05 \mathrm{~g}, \mathrm{NaCl} 0.05 \mathrm{~g}, \mathrm{FeSO}_{4} \cdot 7 \mathrm{H}_{2} \mathrm{O} 0.001 \mathrm{~g}$, agar $1.8 \mathrm{~g}$, deionized water $100 \mathrm{~mL}, \mathrm{pH}$ 7.4-7.6; the medium for fungi is named Martin-Bangladesh red medium, including Rose Bengal agar $3.5 \mathrm{~g}$, deionized water $100 \mathrm{~mL}$; the medium for bacteria is named beef extract peptone agar medium including beef extract $(0.3 \%)$, peptone $(0.5 \%)$ and agar $(1.5 \%)$ in water. Root morphology was measured by CI600 . Fine roots were cleared in $10 \% \mathrm{KOH}$, stained with $0.05 \% \mathrm{w} / \mathrm{v}$ trypan blue for $24 \mathrm{~h}$, and then were cut into 1.5 cm-long segments (Phillips and Hayman 1970). Mycorrhizal infection rate was measured by the glass slide method. Mycorrhizal infection $\operatorname{rate}(\%)=$ formation of twigs of the number of root segments/number of the measured root segments $\times 100$.

Soil $\mathrm{pH}$ and electrical conductivity (Ec) (soil:deionized water 1:5) were determined with a $\mathrm{pH}$ meter and electrical conductivity meter, respectively. Soil acid phosphatase 
activity was measured by the improved method of Tabatabai and Brimner (Zhao and Jiang 1986). Available phosphorus by the $\mathrm{NaHCO}_{3}$ extraction colorimetric method (Bao 1998).

\subsection{Statistical analysis}

All data were subjected to one-way analysis of variance using SPSS program. Means were compared by Duncan's test at the $5 \%$ level.

\section{Results}

\subsection{Plant growth and root morphology}

The impact of different Glomus species on the plant growth of Amygdalus pedunculata Pall. under field conditions is shown in Fig. 1. Compared with the non-inoculated control treatment, the plant height of single inoculation of R.i, single inoculation of F.m, co-inoculation of F.m and R.i increased by $40 \%, 21 \%, 19 \%$ respectively, and the plant crown width increased by $26 \%, 10 \%, 11 \%$. Analysis of variance showed that single inoculation of R.i and single inoculation of F.m significantly increased plant height compared to the control treatment. Single inoculation of R.i proved better than the single inoculation of F.m and the coinoculation of F.m and R.i, while there was no significant difference between single inoculation of F.m and the coinoculation of F.m and R.i. There were no difference in the crown width between inoculated and non-inoculated control treatment. The data indicated that AM fungi can enhance plant growth of Amygdalus pedunculata Pall., in favor of revegetation in the mining subsidence area for ecological recovery.

The impact of different Glomus species on the root growth of Amygdalus pedunculata Pall. is shown in Table 1. Compared with the non-inoculated control treatment, single inoculation of R.i and single inoculation of F.m increased the root projected area and the number of root tips significantly, and single inoculation of R.i increased the root surface area significantly, while there was no significant difference in the average root diameter and volume.

\subsection{Mycorrhizal colonization rate}

Mycorrhizal colonization rate of different treatments for Amygdalus pedunculata Pall. is shown as Fig. 2. The average percentage of root colonization of three treatments was $78 \%$, higher than that of the non-inoculated control treatment (59\%), indicating that all the three Glomus species can increase root colonization. Among the three inoculation treatments, the percentage of root colonization by the single inoculation of F.m was the highest (91\%), and the combined inoculation of F.m and R.i was the second (75\%), and the single inoculation of R.i was the lowest $(67 \%)$.

\subsection{Soil water content}

Soil water content in different treatments was shown in Fig. 3. One year after inoculation, soil water content was not significantly affected. The soil water content of single inoculation of F.m, single inoculation of R.i, and the coinoculation of F.m and R.i was 6.64\%, 5.97\%, 5.38\% respectively, higher than that of the non-inoculated control treatment $(4.71 \%)$, indicating that three inoculation treatments all had certain effect on increasing soil moisture, probably due to the increasement of soil organic matter and soil water capacity. There was no significant difference between these treatments.

\subsection{Soil pH and soil electrical conductivity}

Soil $\mathrm{pH}$ in different treatments for Amygdalus pedunculata Pall. after inoculation 1 year is shown in Fig. 4. There were some differences for different Glomus species, that is the soil $\mathrm{pH}$ of single inoculation of R.i and the co-inoculation of F.m and R.i were both higher than that of the noninoculated control treatment, while the soil $\mathrm{pH}$ of single inoculation of F.m was lower than that of the non-
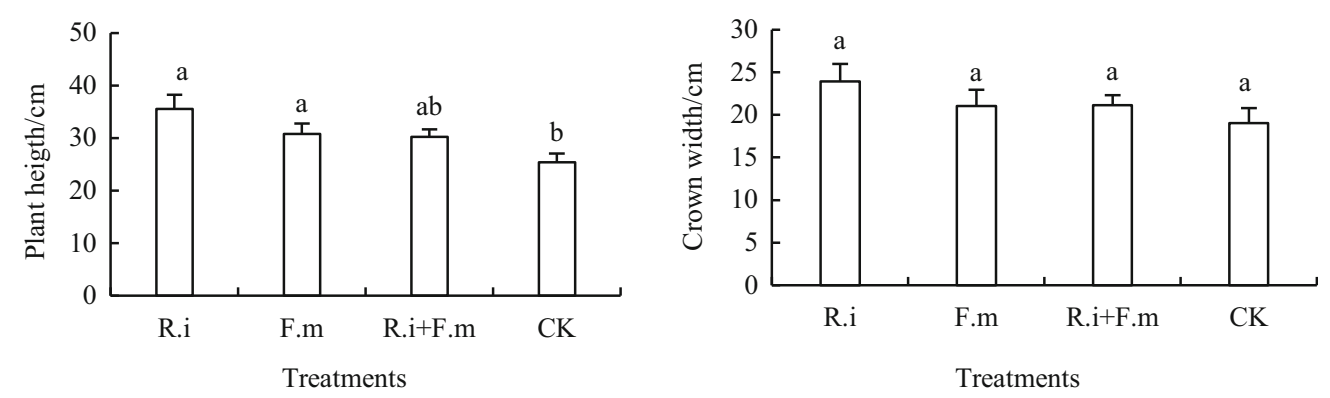

Fig. 1 Effect of AM inoculation on plant height and crown width of Amygdalus pedunculata Pall. 
Table 1 Effect of AM inoculation on root morphology of Amygdalus pedunculata Pall.

\begin{tabular}{lllllll}
\hline Treatment & $\begin{array}{l}\text { Total root length } \\
(\mathrm{cm})\end{array}$ & $\begin{array}{l}\text { Total projected area } \\
\left(\mathrm{cm}^{2}\right)\end{array}$ & $\begin{array}{l}\text { Root surface area } \\
\left(\mathrm{cm}^{2}\right)\end{array}$ & $\begin{array}{l}\text { Average root diameter } \\
(\mathrm{mm})\end{array}$ & $\begin{array}{l}\text { Root volume } \\
\left(\mathrm{cm}^{3}\right)\end{array}$ & $\begin{array}{l}\text { Number of root } \\
\text { tips }\end{array}$ \\
\hline R.i & $182.94 \mathrm{a}$ & $4.25 \mathrm{a}$ & $13.34 \mathrm{a}$ & $0.27 \mathrm{a}$ & $0.11 \mathrm{a}$ & $107 \mathrm{a}$ \\
F.m & $210.45 \mathrm{a}$ & $7.85 \mathrm{a}$ & $19.53 \mathrm{ab}$ & $0.29 \mathrm{a}$ & $0.21 \mathrm{a}$ & $178 \mathrm{a}$ \\
R.i + F.m & $188.56 \mathrm{ab}$ & $4.03 \mathrm{a}$ & $12.65 \mathrm{ab}$ & $0.27 \mathrm{a}$ & $0.10 \mathrm{a}$ & $78 \mathrm{ab}$ \\
CK & $121.80 \mathrm{~b}$ & $3.15 \mathrm{~b}$ & $9.89 \mathrm{~b}$ & $0.26 \mathrm{a}$ & $0.07 \mathrm{a}$ & $92 \mathrm{~b}$ \\
\hline
\end{tabular}

Note Dates are expressed as mean of ten replicates, and different letters in each variable denote means that are significantly different $(P<0.05)$

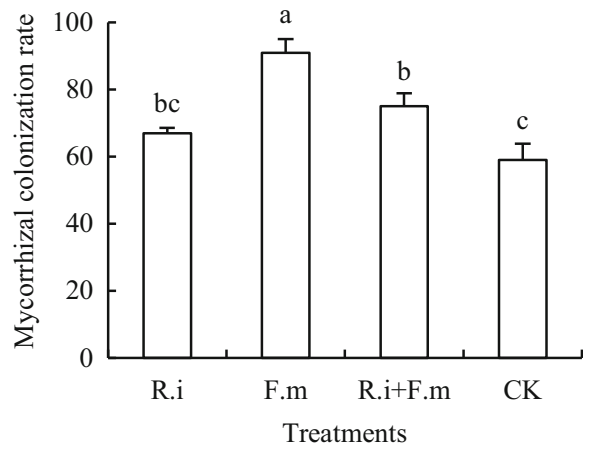

Fig. 2 Effect of AM inoculation on mycorrhizal colonization rate of Amygdalus pedunculata Pall.

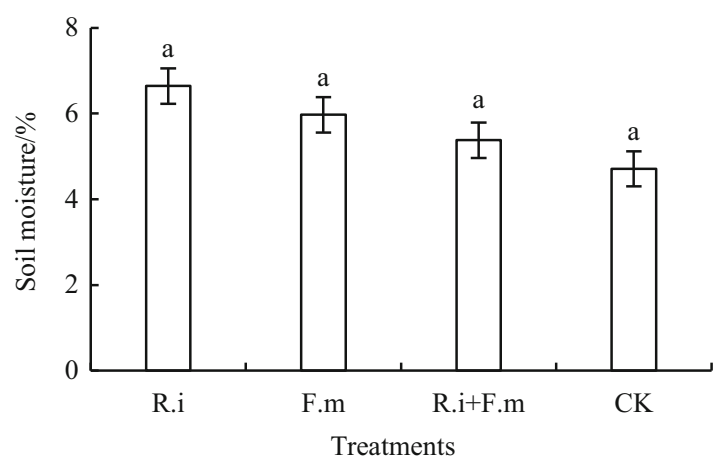

Fig. 3 Effect of AM inoculation on soil moisture of Amygdalus pedunculata Pall.

inoculated control treatment; there was no significant difference between single inoculation of R.i and the coinoculation of F.m and R.i. The electrical conductivity for different treatments is shown in Fig. 4. We can see that all

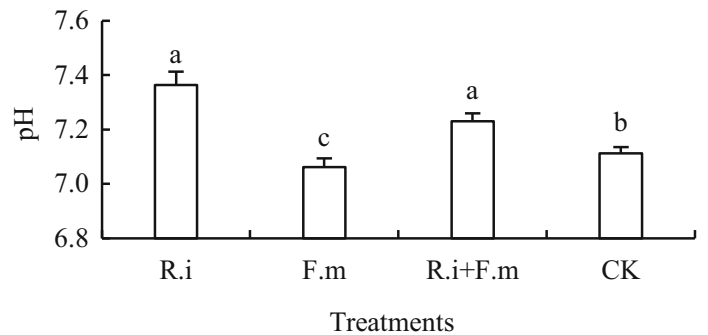

the three inoculation treatments were higher than the noninoculated control treatment, indicating that inoculating AM fungi can increase the soil electrical conductivity.

\subsection{Soil available phosphorus and acid phosphatase}

The effect of different treatments on phosphorus and acid phosphatase activity are shown in Fig. 5. Phosphorus content of all the three inoculation treatments were higher than that of the non-inoculated treatment, indicating that inoculating AM fungi can increase soil available phosphorus, which is vital to the growth and health of plants, and favors the vegetation reconstruction in mining subsidence area. Soil acid phosphatase plays an important role in the transformation and bioavailability of organic phosphorus into inorganic phosphorus. The activity of soil acid phosphatase for different treatment is shown in Fig. 5. The activities of phosphatase of all the three inoculation treatments showed higher than the non-inoculated treatment.AM fungi accelerated the activities of phosphatase in soil rhizosphere, which can promote the transformation of soil insoluble phosphorus into plant available phosphorus, increasing soil available phosphorus.

\subsection{Soil microbe populations}

The effect of different treatments on soil microbe populations was shown in Fig. 6. The quantity of bacteria and fungi of all the three inoculation treatments tended to be higher than that of the non-inoculated control treatment, indicating that AM fungi increased the quantity of bacteria and fungi, especially bacteria. The population of different

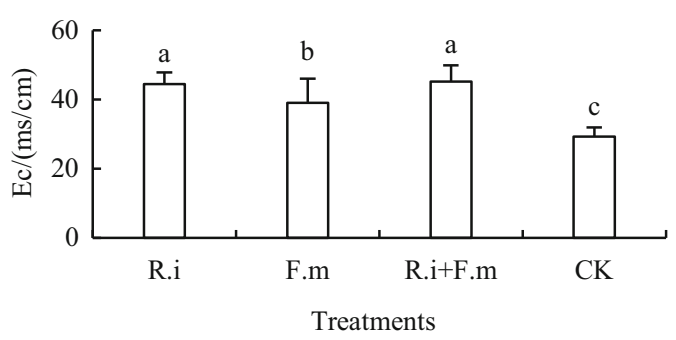

Fig. 4 Effect of AM inoculation on soil pH and Ec of Amygdalus pedunculata Pall. 

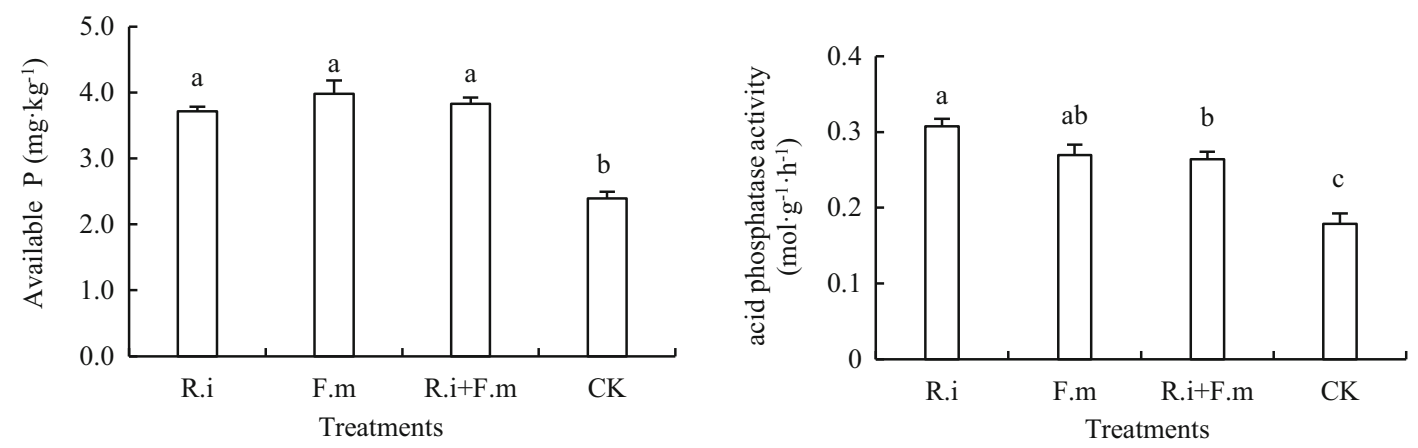

Fig. 5 Effect of AM inoculation on soil acid phosphatase and available phosphorus of Amygdalus pedunculata Pall.
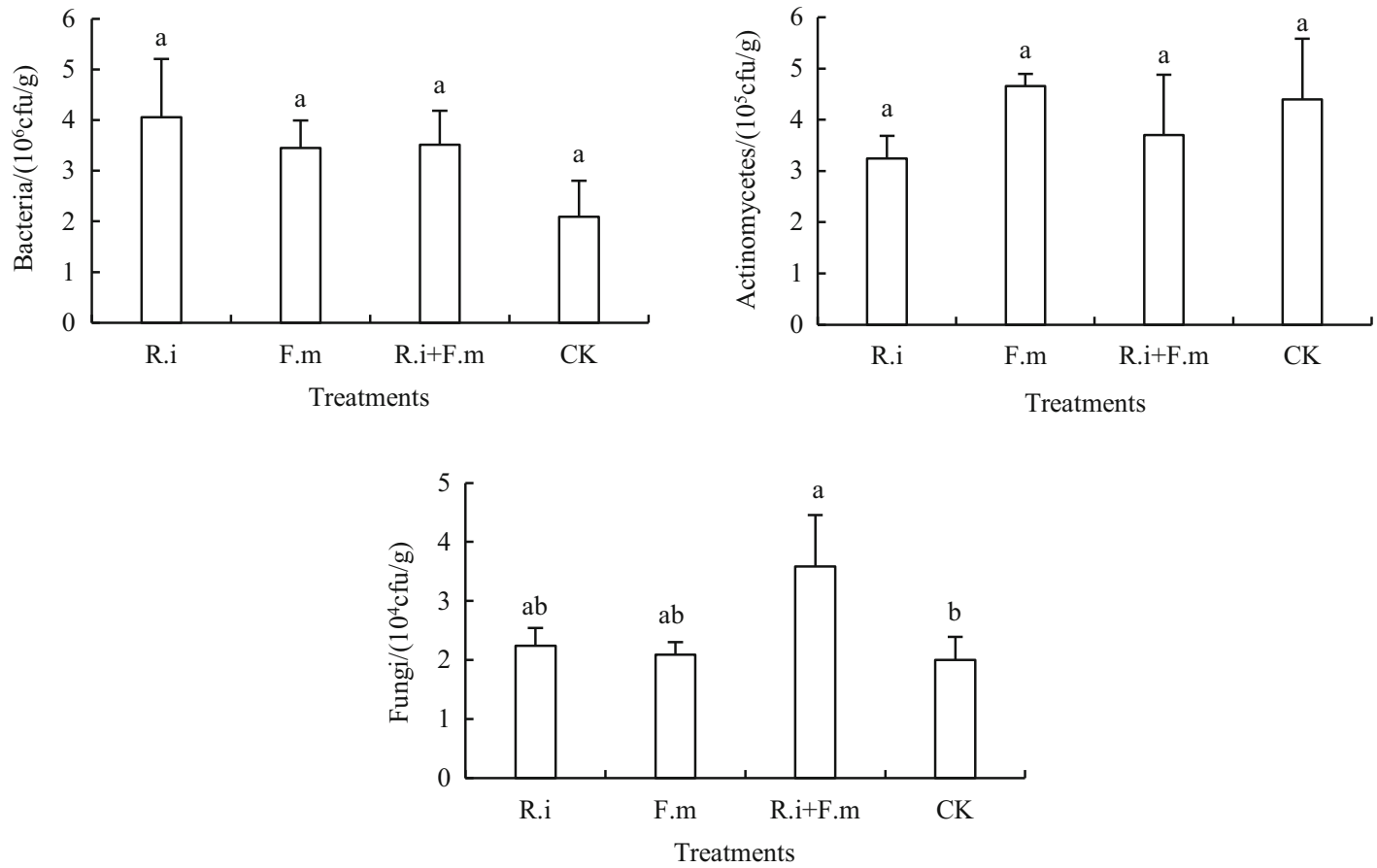

Fig. 6 Effect of AM inoculation on soil microbe populations of Amygdalus pedunculata Pall.

soil microbes tended to decrease in the following order: bacteria (about $80 \%$ of the total number of microbes) $>$ actinomycetes (about $10 \%-20 \%$ of the total number of microbes) $>$ fungi (about $0.2 \%-0.7 \%$ of the total number of microbes). The study area was located at southeast Mu Us desert with poor soil quality, the increase in soil bacteria and fungi can improve the soil microbial and nutritional environment, conducive to the plant growth and local ecology recovery.

\section{Discussion}

Mycorrhizas are symbiotic associations established between certain soil fungi and most vascular plants. They play a good role in optimizing plant fitness and soil quality
(Barea et al. 2011). In this study, different kinds of fungi species could infect plant roots of Amygdalus pedunculata Pall., which indicate that good symbiosis between AM fungi and Amygdalus pedunculata Pall. has been formed. Mycorrhizal colonization rate of different treatments were higher than that of the non-inoculated control treatment, which showed artificial inoculation to be effective in increasing root mycorrhizal colonization. In addition, the infection rate of different treats were different, showing that host plant and fungi select each other and its colonization rate may be probably influenced by host plants and AM fungal isolates.

Mycorrhizal symbiosis could improve the resilience of plant communities against environment stresses, including nutrient deficiency, drought and soil disturbance and promote the growth rate of the plant. After inoculation for 
1 year, plant height of inoculation treats presented significantly higher than that of control treatment. At the same time, certain indicators of the roots of inoculated plant were higher than that of no-inoculated plant. Rapid growth of plant is beneficial to the local ecological restoration, especially for ecological fragile mining areas. These results are consistent with former research (Wang et al. 2014).

Water is a key factor of plant growth, especially for the arid mining area where water loss is serious. Mycorrhiza can enhance nutrient acquisition and water absorption of host plants by extending hyphae beyond the nutrient depletion zone of roots (Wu et al. 2015). Through the development of hyphae network from mycorrhizal roots into the surrounding soil, hyphae directly enmesh soil particles. Besides AMF could release glomalin-related soil protein (GRSP) into soils, which could improve soil structure and improve water retention of rhizosphere (Bedini et al. 2009; Matthiasc et al. 2010). In this study, rhizosphere soil moisture content increase than control, probably due to the increase of soil organic matter and soil water holding capacity. After mycorrhizal inoculation, plant rhizosphere environment has also changed soil $\mathrm{pH}$ value and conductivity changed after mycorrhizal inoculation for 1 year, which may be due to the secretion caused a certain effect on rhizosphere soil biochemical reaction after mycorrhizal inoculation. Meanwhile, the content of available $\mathrm{P}$ for different inoculation treatments showed higher than that of non-inoculated treatment, which may be the result of higher soil acid phosphatase activities, which could promote the transformation of soil insoluble phosphorus into plant available phosphorus.

Microorganisms in the soil are critical in decomposing organic residues and recycling soil nutrients, and is one of the indicators of soil quality evaluation. Soil microorganism quantity and enzyme activity changes because of mining activities (Quadros et al. 2016). Arbuscular mycorrhizal inoculation can significantly improve the survival environment of rhizosphere microbes, is conducive to the improvement of the quality of the soil and the growth of plants. Research conclusions of our study were consistent with Camilla (Camilla et al. 2003).

\section{Conclusion}

Coal mining subsidence causes numerous environmental problems which have negative effects on vegetation growth. In this study, we found that AMF inoculation could promote the shoot growth and the root development of Amygdalus pedunculata Pall. The AMF inoculated plant showed higher root mycorrhizal colonization, soil acid phosphatase and available phosphorus as well as soil electrical conductivity compared to control. Single inoculation of R.i had good performance in plant height, soil moisture, and soil microbe population quantity; while single inoculation of F.m had good performance in soil available phosphorus concentration and soil fungi quantity. Associated inoculation of F.m and R.i can increase the soil electric conductivity and enhance soil acid phosphatase activity. AMF inoculation can be effective method for further ecological restoration in fertile environment in coal mine subsided areas.

Acknowledgements The study was financially supported by the National Natural Science Foundation of China (51574253) and the National Key Research and Development Program of China (2016YFC0501106).

Open Access This article is distributed under the terms of the Creative Commons Attribution 4.0 International License (http://crea tivecommons.org/licenses/by/4.0/), which permits unrestricted use, distribution, and reproduction in any medium, provided you give appropriate credit to the original author(s) and the source, provide a link to the Creative Commons license, and indicate if changes were made.

\section{References}

Bao SD (1998) Soil agricultural chemistry analysis. China Agriculture Press, Beijing, pp 56-83

Barea JM, Palenzuela J, Cornejo P, Sánchezcastro I, Navarrofernández C, Lopézgarcía A (2011) Ecological and functional roles of mycorrhizas in semi-arid ecosystems of southeast spain. J Arid Environ 75(12):1292-1301

Bedini S, Pellegrino E, Avio L, Pellegrini S, Bazzoffi P, Argese E et al (2009) Changes in soil aggregation and glomalin-related soil protein content as affected by the arbuscular mycorrhizal fungal species Glomus mosseae and Glomus intraradices. Soil Biol Biochem 41(7):1491-1496

Bi YL, Zhang YX (2014) Role of the different planting age of seabuckthorn forests to soil amelioration in coal mining subsidence land. Int J Coal Sci Technol 1(2):192-197

Camilla W, Søren Christensen I, Jakobsen AK Müller, Sørensen SJ (2003) The mycorrhizal fungus (Glomus intraradices) affects microbial activity in the rhizosphere of pea plants (Pisum sativum). Soil Biol Biochem 35(10):1349-1357

Cao ZW, Wang C, Zhang JB, Bi GY, Bi Y, Wen BY, Li WY, Li YX (2016) Growth of seedlings and morphological characteristics of leaves for different provenance of introduced Amygdalus pedunculata. Prot For Sci Technol 158(11):1-3

Chandanie WA, Kubota M, Hyakumachi M (2009) Interactions between the arbuscular mycorrhizal fungus Glomus mosseae and plant growth-promoting fungi and their significance for enhancing plant growth and suppressing damping-off of cucumber (Cucumis sativus 1.). Appl Soil Ecol 41(3):336-341

Chen Q, Li C, Fang ZG, Chen B, Shen YH (2013) Preparation of biodiesel from desert Amygdalus pedunculata pall oil. J Northwest Univ (Nat Sci Edn) 43(2):229-232

del Carmen Jaizme-Vega M, Rodríguez-Romero AS, Núñez LAB (2006) Effect of the combined inoculation of arbuscular mycorrhizal fungi and plant growth-promoting rhizobacteria on papaya (Carica papaya 1.) infected with the root-knot nematode Meloidogyne incognita. Fruits 61(3):151-162

Gong MG, Tang M, Zhang QM, Feng X (2012) Effects of climatic and edaphic factors on arbuscular mycorrhizal fungi in the 
rhizosphere of Hippophae rhamnoides, in the loess plateau, china. Acta Ecol Sin 32(2):62-67

Latef AAHA, He CX (2011) Effect of arbuscular mycorrhizal fungi on growth, mineral nutrition, antioxidant enzymes activity and fruit yield of tomato grown under salinity stress. Sci Hortic 127(3):228-233

Li W, Chen LQ, Zhou TJ, Zhang K, Long LI (2011) Research progress of soil quality in china mining subsidence area. Coal Sci Technol 05:125-128

Ma XW, Guo CH, Luo M (2006) Influence of endocarp, salt and water stress on the seed germination of Amygdalus pedunculata. J Northwest For Univ 21(4):69-72

Matthiasc R, Noorf M, Evaf L, Pedrom A (2010) Mycelium of arbuscular mycorrhizal fungi increases soil water repellency and is sufficient to maintain water-stable soil aggregates. Soil Biol Biochem 42(7):1189-1191

Miller RM, Jastrow JD (1992) The role of mycorrhizal fungi in soil conservation. ASA Special Publication, Madison, pp 29-44

Phillips JM, Hayman DS (1970) Improved procedures for clearing roots and staining parasitic and vesicular-arbuscular mycorrhizal fungi for rapid assessment of infection. Trans Br Mycol Soc 55(1):158-161

Quadros PDD, Zhalnina K, Davis-Richardson AG, Drew JC, Menezes FB, Camargo FADO et al (2016) Coal mining practices reduce the microbial biomass, richness and diversity of soil. Appl Soil Ecol 98:195-203

Shen P, Fan XR, Li GB (1999) Microbiology experiment. Higher Education Press, Beijing, pp 123-128
Tian Y, Zhang HH, Meng XY, Wang J, Hu JW, Sun GY (2013) Effects of arbuscular mycorrhizal fungi (Glomus mosseae) on growth and photosynthesis characteristics of Lolium merenne L. under Cd contaminated soil. Acta Agrestia Sin 21(1):135-141

Tripathi N, Singh RS, Singh JS (2009) Impact of post-mining subsidence on nitrogen transformation in southern tropical dry deciduous forest, India. Environ Res 109(3):258-266

Wang JG, Gao XM, Bai SL, Zheng R, Liu M (2014) Effects of arbuscular mycorrhiza on the drought tolerance of Prunus mongolica. J Arid Land Resour Environ 12:138-142

Wu QS, Xia RX, Zou YN (2008) Improved soil structure and citrus growth after inoculation with three arbuscular mycorrhizal fungi under drought stress. Eur J Soil Biol 44(1):122-128

Wu QS, Srivastava AK, Cao MQ, Wang J (2015) Mycorrhizal function on soil aggregate stability in root zone and root-free hyphae zone of trifoliate orange. Arch Agron Soil Sci 61(6):813-825

$\mathrm{Xu} \mathrm{XQ}$, Chu JM (2013) Analysis on development potential and countermeasures of Amygdalus pedunculata industry. For Resour Manag 2(1):22

Zang YT, Wang J, Ding GD, Gao Y, He X, Yan L, He Z, Na Q, Gong P, Ren Y (2010) Variation of physico-chemical properties of aeolian sandy soil at coal mining subsidence and its evaluation. Acta Pedol Sin 47(2):262-269

Zhao LP, Jiang Y (1986) Determination of the method of soil phosphatase activity. Chin J Soil Sci 17(3):138-142 